\title{
Hippocampal Neurons in the Monkey with Activity Related to the Place in Which a Stimulus Is Shown
}

\author{
E. T. Rolls, Y. Miyashita, ${ }^{a}$ P. M. B. Cahusac, R. P. Kesner, ${ }^{b}$ H. Niki, ${ }^{c}$ J. D. Feigenbaum, and L. Bach \\ Department of Experimental Psychology, University of Oxford, Oxford OX1 3UD, England
}

In order to analyze the functions of the hippocampus in the primate, and to advance the understanding of amnesia, the activity of 994 single hippocampal neurons in the monkey was analyzed during the performance of a task known to be affected by hippocampal damage in which both an object, and its position in space, must be remembered. The serial multiple object-place memory task required a memory for the position on a video monitor in which a given object had appeared previously. It was found that $9.3 \%$ of neurons recorded in the hippocampus and hippocampal gyrus had spatial fields in this and related tasks, in that they responded whenever there was a stimulus in some but not in other positions on the screen. We found that $2.4 \%$ of the neurons responded to a combination of spatial information and information about the object seen, in that they responded more the first time a particular object was seen in any position. Six of these neurons were found that showed this combination even more clearly, in that, for example, they responded only to some positions and only if it was the first time that a particular stimulus had appeared there.

It is concluded that there are neurons in the primate hippocampus which (1) respond to position in space and (2) in some cases combine information about stimuli and their position in space, responding to a stimulus only the first time it is seen in a position in space, for example. Thus, not only is spatial information processed by the primate hippocampus, but it can be combined with information about which stimuli have been seen before. The ability of the hippocampus to form such conjunctions may be an important property for its role in memory.

Bilateral damage to the temporal lobe in humans can cause anterograde amnesia (Scoville and Milner, 1957; Milner, 1982). A number of structures are damaged, and these include the hippocampus and the amygdala. Experimental investigations have been performed to determine which structures are crucial in producing the amnesia and to analyze the neural bases of the

\footnotetext{
Received Apr. 16, 1987; revised Aug. 24, 1988; accepted Scpt. 19, 1988.

This work was supported by the Medical Research Council.

Correspondence should be addressed to Dr. E. T. Rolls, Department of Exper imcntal Psychology, University of Oxford, South Parks Road, Oxford OX1 3UD, England.

a Present address: Department of Physiology, University of Tokyo, Bunkyo-ku, Tokyo, Japan.

b Present address: Department of Psychology, University of Utah, Salt Lake City, Utah 84112 .

'Present address: Department of Psychology, I Iniversity of Tokyo, Runkyo-ku Tokyo, Japan.

Copyright (C) 1989 Society for Neuroscience $0270-6474 / 89 / 061835-11 \$ 02.00 / 0$
}

different types of amnesia (Squire and Zola-Morgan, 1983). It has been shown that the monkey's ability to remember a list of objects in a recognition task is impaircd by combined damage to the hippocampus and amygdala but is much less affected by damage to the hippocampus or amygdala alone (Mishkin, 1978). In analyzing the way in which the hippocampus could contribute to a memory deficit, it has been shown that tasks which are particularly affected by hippocampal damage in the primate include those in which both an object and the place in which it was seen must be remembered (Mishkin, 1982; Gaffan and Saunders, 1985; Gaffan, 1987; Parkinson et al., 1988). It is thus of interest that H.M., and humans with right temporal lobe damage, exhibited impaired memory of the positions in which objects had been placed on a board (Smith and Milner, 1981).

In analyses of the functions of the hippocampus in the rat, it has been suggested that rats with hippocampal damage have an impaired ability to make a map of space, in that they are impaired in running correctly in an 8-arm maze, or in swimming correctly to a submerged platform, in situations in which cxtramaze cues must be used to restitute their position in space (O'Keefe and Nadel, 1978; Morris et al., 1982). There is evidence that in the rat some hippocampal neurons fire when the animal is in a particular place in an environment (O'Keefe, 1979, 1983). It is thought that the place at which a cell fires is defined by the spatial configuration of the cues in the environment. Different cells respond when the rat is in different places in space. An alternative concept of the function of the hippocampus in the rat suggests that the hippocampus is involved in working memory, on the basis of deficits in both nonspatial and spatial short-term memory tasks in rats with hippocampal lesions (Olton et al., 1979; Olton, 1983).

In order to analyze the functions of the hippocampus in the primate and to advance the understanding of amnesia, we are recording the activity of single hippocampal neurons in the monkey during the performance of tasks known to be affected by hippocampal damage. In this paper we describe the activity of single neurons in a task in which the monkey must remember whether an object has been seen before in a particular place. Object-place memory tasks are one type of memory task that has been shown to be particularly impaired by hippocampal (but not by amygdala) damage in the monkey (Mishkin, 1982; Gaffan and Saunders, 1985; Gaffan, 1987). The multiple objectplace memory task used here required a memory of 4 or 9 positions on a television screen where a given object had appeared previously, and thus was quite demanding. It was found in this task that some hippocampal neurons responded best when an object appeared in one or several positions as compared with other positions on the screen. In the only previous record- 
ings we know from the hippocampus of the behaving monkey, it has been shown that some hippocampal neurons are active in a delayed match to sample task (Brown, 1982) and that some hippocampal neurons are active in a delayed response task (Watanabe and Niki, 1985; Cahusac et al., 1989). A further aim of the experiments described here was to investigate the activity of hippocampal neurons in a serial recognition task to determine whether the responses of any hippocampal neurons are related to whether stimuli have been seen recently, as the hippocampus at least contributes (as does the amygdala) to this form of memory. The advantage of using a serial recognition task is that it allows the number of intervening stimuli over which neuronal responses reflect familiarity to be measured (Rolls et al., 1982).

\section{Materials and Methods}

Serial multiple object-place memory task. In the serial object-place memory task, the monkey had to remember the particular positions in which he had seen a particular stimulus previously. On each trial, a stimulus was shown in 1 of 4 or in 1 of 9 positions on a video monitor. (In the 4-position task, the positions were arranged in 2 rows of 2 columns, with position 0 in the top left and position 3 in the bottom right. In the 9-position task, the positions were arranged in 3 rows of 3 columns, with position 0 in the top left and position 8 in the bottom right.) Each stimulus was shown in each position twice. The first time a stimulus was shown in a particular position, the monkey had to avoid licking a tube in front of his mouth, in order to avoid obtaining a taste of saline. The second time the monkey saw the stimulus in a given position, he could lick the tube to obtain fruit juice. For each new stimulus, the computer randomized the order of the positions in which a stimulus was shown, subject to the constraint that the same reinforcement contingency should not be repeated consecutively more than 3 times. After each stimulus had been shown in each position twice, the computer continued the task with a new stimulus. (Some of the stimuli had been used on previous days, but once a sequence of novel/familiar positions had been run with a given stimulus on a particular day, that stimulus was not used again on that day.) The task allowed an object to be shown 6 times in other positions before it reappeared in the same position in the $2 \times 2$ version, and up to 16 intervening trials in the 3 $\times 3$ version. In a spatial response version of the $2 \times 2$ task the monkey was trained to reach and touch the quadrant where the stimulus appeared instead of licking at a tube on positively rewarding trials. A simpler spatial response version (touch-position task) was also used in which the same stimulus appeared on all trials and the monkey could reach and touch the relevant quadrant to receive a reward for each touch. The latter 2 spatial response tasks were tested in 2 monkeys. However, as some of the results were similar for all 3 tasks, some of the data are analyzed together. The tasks were completely computer-controlled to ensure that no influence by the experimenters on the monkey's behavior or on the neuronal activity was possible. The computer switched the stimuli on and off for each trial, and synchronized its data collection so that the stimulus was turned on at the start of the 21 st bin of a peristimulus time histogram. The stimuli were displayed $30 \mathrm{~cm}$ from the monkey on a color video monitor subtending $12^{\circ}$ at the retina and occupying the appropriate quarter or ninth of the monitor screen. The stimuli were either junk pseudo-colored stimuli digitized from the television or were simple geometric shapes such as boundary curvature descriptors (Schwartz et al., 1983). The resolution of these images was $256 \times 256$ pixels with 256 gray levels or colors. The stimuli were stored on the PDP11 computer disk ready for random access loading into an AED512 video framestore.

The monkeys were also trained to perform a visual discrimination task, which was run as a control to ensure that any results obtained were not due to obtaining reinforcement, licking for fruit juice, etc. If a circle, the positive discriminative stimulus $(S+)$, appeared on the monitor, the monkeys could lick to obtain a fruit juice reward, and if a square of the same area and luminance, the negative discriminative stimulus ( $\mathrm{S}-$ ), appeared the monkey had to withhold licking in order to avoid aversive hypertonic saline.

A $0.5 \mathrm{sec}$ signal tone $(400 \mathrm{~Hz})$ preceded the presentation of the stimulus in all tasks, and if the monkey was fixating correctly before the stimulus appeared, he had sufficient time to perform the discrimination and obtain multiple licks of the fruit juice tube in the short $(1.0 \mathrm{sec})$ period in which the stimulus was on. This procedure was designed to ensure fixation of the stimuli (Rolls et al., 1979). The order of presentation of the stimuli was randomized. The EOG recordings, and recordings made in one monkey with the search coil method (Judge et al., 1980), confirmed that this procedure resulted in consistent fixation of the stimuli.

The monkeys were also trained to perform a serial recognition task (Rolls et al., 1982), in order to determine whether neurons active in the serial multiple object-place memory task might be responsive on the basis of the novelty as compared to the familiarity of visual stimuli, independently of any spatial component to the task. The serial recognition task was an automated version of one used previously (Rolls et al., 1982). Each stimulus was presented twice a day, once as novel and once as familiar. When a stimulus was novel, the monkey had to refrain from licking in order to avoid the saline liquid. 'When a stimulus was familiar, the monkey could obtain fruit juice by licking the tube in front of its mouth. Up to 17 other stimuli intervened between the novel and familiar presentations of a given stimulus. The stimuli were similar to those used in the object-place serial memory task, except that they filled the monitor screen.

Recording techniques. The activity of single neurons was recorded with glass-insulated tungsten microelectrodes (after Merrill and Ains:worth, 1972, but without the platinum plating) in 3 macaque monkeys (Macaca mulatta) (weight, $3.0-4.5 \mathrm{~kg}$ ) seated in a primate chair using techniques that have been described previously (Rolls et al., 1976). The monkeys had been implanted under thiopentone sodium anesthesia with stainless-steel holders on which an adaptor could be fitted for the later daily recording sessions. The action potentials of single cells were amplified using techniques described previously (Rolls et al., 1979), converted into digital pulses using the trigger circuit of an oscilloscope, and analyzed on-line using a PDP11 computer. The computer collected peristimulus rastergrams of neuronal activity for each trial and displayed, printed, and stored each trial, as well as computing the peristimulus time histogram by summing trials of a given type. To facilitate latency measurements, the cumulative sum distribution was calculated from the sum peristimulus time histogram. For each trial the number of action potentials occurring in a $500 \mathrm{msec}$ period (and a $250 \mathrm{msec}$ period) starting $100 \mathrm{msec}$ after the stimulus onset was printed. This period was chosen because the neurons studied responded to visual stimuli with latencies that were typically $100 \mathrm{msec}$ or more, and the monkeys consistently fixated the stimuli for more than $500 \mathrm{msec}$. Fixation of the stimuli was confirmed using permanently implanted silver/ silver chloride electrodes for EOG recording. The EOG recordings provided eye position to within $1^{\circ}-2^{\circ}$ and were sampled by the computer every $10 \mathrm{msec}$ and saved with the action potentials for each trial.

Radiographs were used to locate the position of the microelectrode on each recording track relative to permanently implanted reference electrodes and bony landmarks such as the posterior tip of the sphenoid bone (Aggleton and Passingham, 1981). Cell positions were reconstructed from the $X$-ray coordinates taken together with serial $50 \mu \mathrm{m}$ histological sections stained with cresyl violet which showed the reference electrodes and microlesions made at the end of some of the microelectrode tracks.

Treatment of results. Measures of responses for each cell were calculated from the total number of action potentials occurring on each trial in the 100-600 msec period following stimulus onset. This period was chosen because the cells studied typically responded in the task with latencies greater than $100 \mathrm{msec}$ after the onset of the visual stimuli. Recordings of fixation usually confirmed that the monkeys fixated during this period of firing rate measurement.

Analyses of variance were then performed on the responses of each cell to novel and to familiar stimuli shown in each position. If a significant difference between the responses was found in the ANOVA, then subsequent multiple $t$, Tukey, Duncan, and Newman-Keuls analyses (see Bruning and Kintz, 1977) were performed to determine whether the effect was related to the different places on the screen, to novel as compared with familiar stimuli, to an interaction between these, or to the particular stimulus shown. The figures present the mean firing rate and its SE to stimuli shown in each position, typically based on 4-10 presentations of the stimulus. The results of the analysis of variance are also usually indicated. Cells were classified as showing differential responses if the ANOVA was significant at the 0.05 level, but for most of the cells described here with differential responses, the differences were significant at beyond the 0.01 level. A few such significant results 
A
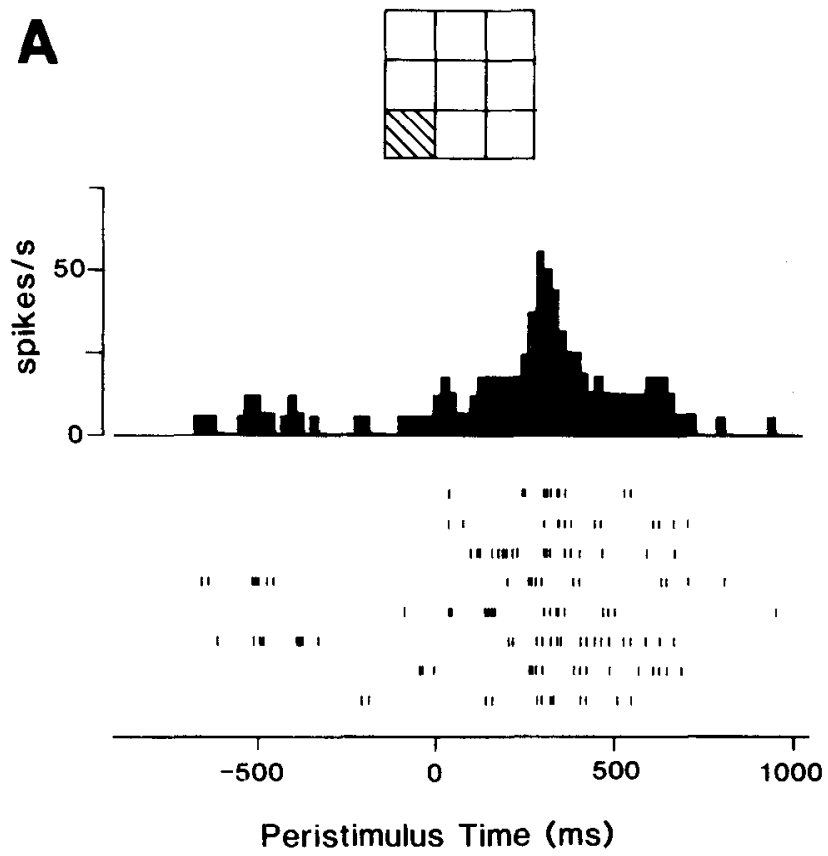
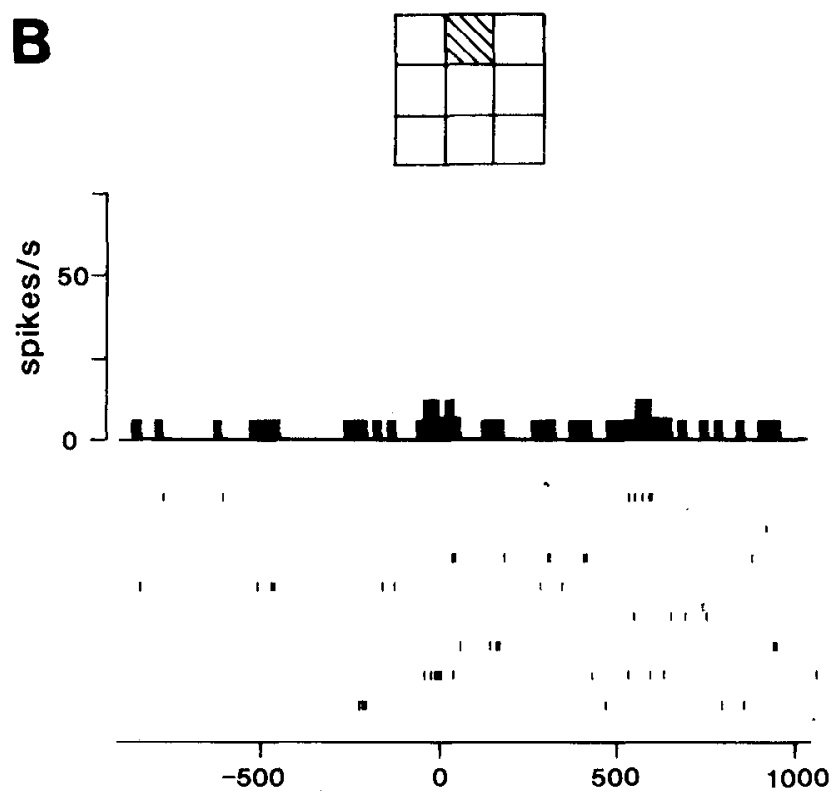

Peristimulus Time (ms)

Figure 1. Rastergrams and peristimulus time histograms of a single hippocampal neuron that responded during the $3 \times 3$ version of the multiple object-place task to stimuli presented to the bottom left-hand corner of the monitor screen (inset above histogram) as shown in $A$. The neuron responded less when the stimulus was presented at the middle top position as shown in $B$. This neuron had a differential spatial latency of 100 msec.

might be expected by chance among the population of neurons from which recordings were made. To test whether more cells had significant results than would be expected by chance, the number of cells with significant results at each levcl (e.g., $p<0.05, p<0.01$, and $p<0.001$ ) found in the ANOVA was compared using a goodness of fit $\chi^{2}$ test with the number that would be predicted by chance.

The latency of neuronal responses, or the differential latency of the neuronal response, that is, the latency at which it fired significantly differently in 2 conditions of the task, such as 2 different stimulus positions, was determined using cumulative sum and running mean statistics. The cumulative sum (Woodward and Goldsmith, 1964) was calculated on-line, using 18 prestimulus bins as the reference. The point at which the slope of the cusum changed was taken as the latency. Running mean $t$ tests, which compared the mean number of neuronal spikes in 18 prestimulus bins with the mean number of spikes in 2,3 , 4 , or 5 poststimulus bins, were performed over the sums of trials of any one condition, over the difference of the sums of trials of 2 conditions, or over the cumulative sums of these arrays of valucs.

\section{Results}

The activity of 994 neurons was recorded during the performance of the object-place memory task in 3 monkeys. Of these neurons, 249 were active in the task. The response properties of the 93 neurons that responded differently (as confirmed by the analyses of variance described in Materials and Methods) when objects were shown in different positions on the screen are described first. The responses of 66 hippocampal neurons that responded in this task consisted of an increase in firing rate from a mean spontaneous level of 12.3 (SD \pm 10.6 ) spikes/sec to 24.7 ( $\mathrm{SD} \pm 14.5)$ spikes/sec. Seventeen neurons decreased their firing from a mean of 28.6 (SD \pm 11.1 ) spikes $/ \mathrm{sec}$ to 15.2 $(\mathrm{SD} \pm 8.2$ ) spikes/sec. The responses of the other neurons that altered their activity during the tasks were generally small, often occurred during the cue tone period, and were nondifferential with respect to any of the parameters such as spatial position and familiarity studied here. The activity of these neurons is not considered further in this paper, but other tasks effective in driving hippocampal neurons are described elsewhere (Cahusac et al., 1989; Miyashita et al., 1989).

Rastergrams and peristimulus time histograms of the activity of a single hippocampal neuron in the multiple object-place memory task are shown in Figure 1. This neuron fired more when an object was shown in position 6 (bottom-left corner) than in position 1 (middle top). The latency of the neuronal response in the task was $100 \mathrm{msec}$, and this was the latency at which the neuron responded significantly differently to objects shown in the different positions, as shown by cusum tests and by running mean statistics.

The responses of 4 neurons in the $2 \times 2$ version of the objectplace memory task are shown in Figure 2, with the mean response and its SE for each position indicated on a diagram of the monitor screen. For each neuron, the ANOVA showed that the neuron responded significantly differently when objects were shown in different positions on the monitor. Some of the neurons responded primarily when objects were shown in one position, and others responded when objects were shown in some, but not in other, positions.

Three neurons were tested in both the $2 \times 2$ and the $3 \times 3$ versions of the object-place memory task. The responses obtained from one neuron tested in this way are shown in Figure 3. For all 3 neurons there was some correspondence between the positions to which a neuron responded in the 2 versions of the task.

The EOG or search coil recordings showed that in these tasks the monkey typically moved his eyes to the position on the screen in which the stimuli were shown within $200 \mathrm{msec}$ of the onset of the visual stimuli. An example is shown in Figure 4. The responses of the neuron shown in Figure 4 occurred when the monkey was looking at an object at one of the positions on the screen. The neuron did not respond in control tests in which the monkey made the same eye movements to fixate a small 


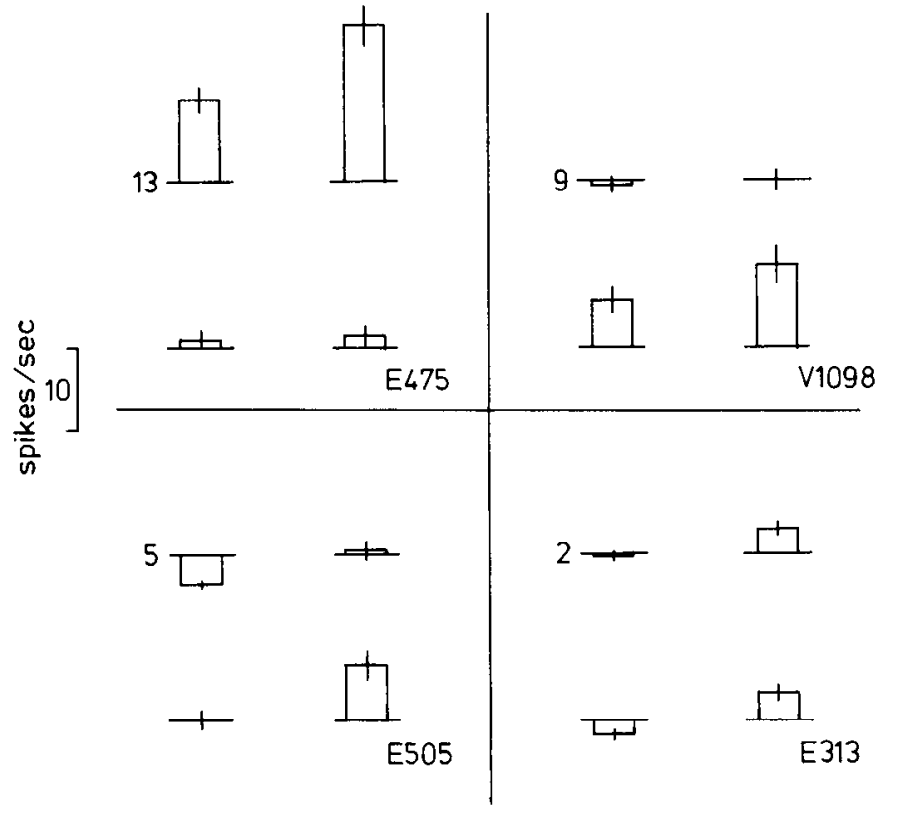

Figure 2. Typical differential spatial responses of 4 hippocampal formation neurons. In each quadrant a different neuron's response is shown. The responses for each of the 4 different positions in which stimuli appear $(2 \times 2$ version of multiple object-place task) are displayed relative to the spontaneous firing baseline level in spikes/second, as indicated by the number beside each top left corner position. The SE bars are indicated for each of the mean responses. For example, cell number E475 responded to the 2 upper positions on the screen and V1098 responded preferentially to the bottom 2 positions.

spot, so that the neuronal responses were due not just to eye movements. (Good fixation of the small spot occurred because the monkey could obtain fruit juice by detecting dimming of the fixation spot. Fixation was confirmed with the eye coil technique.) This visual fixation task with a small spot was repeated for 46 cells with spatial responses, and 39 of these cells had no activity related to the place in which the fixation spot was shown, so that the activity of these 39 cells was not related to eye movements, and did not occur either to a small spot placed in the spatial field of the cells. Instead, these cells required a visual stimulus such as the pictures used in the object-place memory task to be placed in the spatial field in order to respond. Seven of the 46 cells did respond in the visual fixation task, and the activity of these 7 neurons could thus have been related to eye movements or to the appearance of the small spot in the spatial field of the cell.

The latencies of the responses of these neurons are plotted in the upper part of Figure 5. Cumulative sum and running mean statistics were used to estimate the neuronal response latencies. Many of the neurons had response latencies in the range of 100 $200 \mathrm{msec}$, with a mean value for the population (excluding those in the tail of the distribution beyond $260 \mathrm{msec}$ ) of $168 \mathrm{msec}$.

A proportion of the neurons ( $n=24 ; 2.4 \%$ of the total) responded in relation to the relative novelty (or familiarity) component of the object-place memory task. For example, 19 ncurons responded differentially (typically more) the first time a stimulus was seen in each position on the screen compared with the second time the same stimulus was seen in the same position on the screen. (This occurred independently of whether the stimulus had already been seen in other positions on the screen.) Thus, these neurons combined information derived from the
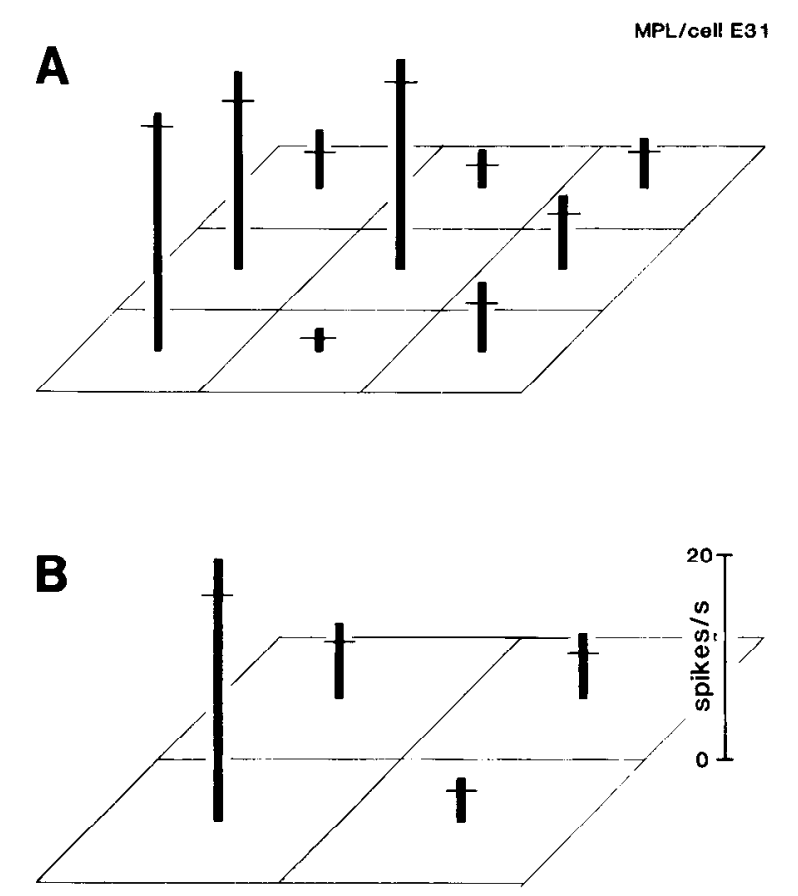

C

spontaneous discharges

Figure 3. Example of the spatial responses of a neuron tested with both the $3 \times 3(A)$ and $2 \times 2(B)$ versions of the multiple object-place task. In the $3 \times 3$ version, the neuron responded to the bottom lefthand corner and middle quadrants of the screen, and a consistent response to those positions was also seen using the $2 \times 2$ version of the task. $C$, Spontaneous firing rate preceding the presentation of stimuli.

stimulus with information about its position. (I'en of these neurons also responded better to some than to other positions on the screen, that is, they had space fields as described abovc, but in addition responded more to novel stimuli than to familiar stimuli in each position in space. Thus, for these neurons in the 2-way ANOVA there were significant effects of both novelty and position on the screen.) In the case of 6 neurons there was an interaction between novelty and position. An example of one such neuron is shown in Figure 6, where a differential novel/ familiar response occurs primarily for the right upper quadrant of the screen (as shown by a very significant interaction term in the ANOVA). An example of another neuron with an interaction term between object and spatial information is shown in Figure 7. Although these 24 neurons that combined information about spatial position and the novelty of the object in that position were not common, and represented $2.4 \%$ of those analyzed, there were many more statistically highly significantly responding such neurons as shown by the ANOVA than would be predicted by chance (as tested by a goodness-of-fit $\chi^{2}$ test: $\chi^{2}$ $=76.3, d f=1, p<0.001)$. The mean response latency of these neurons which combined information about position with information about whether an object had been seen before in that position (excluding neurons with latencies greater than $260 \mathrm{msec}$ as before) was $196 \mathrm{msec}$. The distribution of such latencies is shown in the lower part of Figure 5. It was further noted that 
A
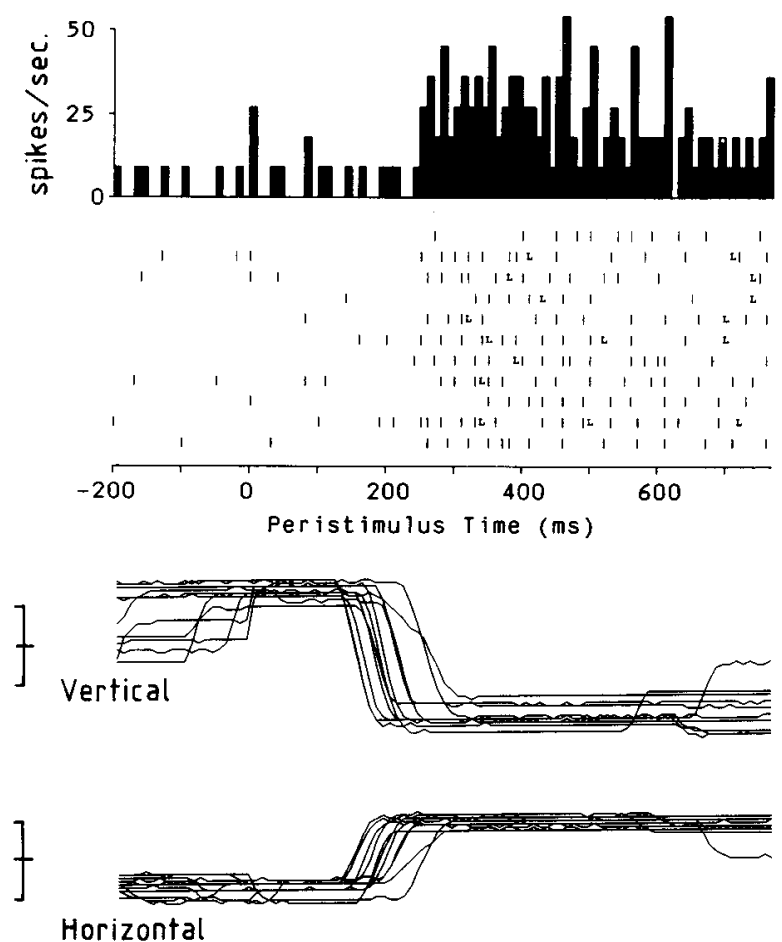

B

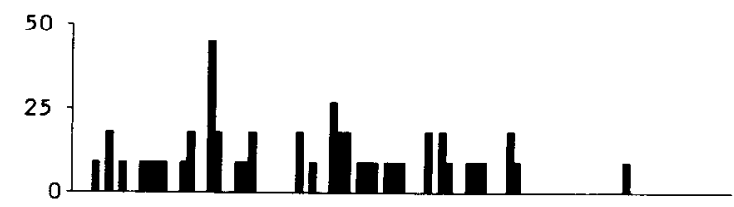

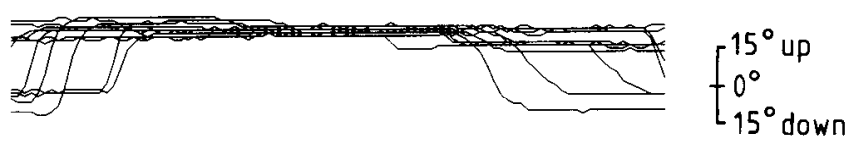

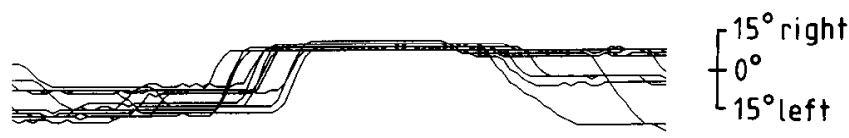

Figure 4. Simultaneous recordings of eye position with the search coil technique (shown below each rastergram) and of the activity of a hippocampal neuron with spatial responses indicate that the monkey typically moved his eyes to fixate the stimuli in the spatial tasks soon after the stimuli appeared (at time 0). This particular neuron responded after the monkey fixated the stimulus when it was shown in one position on the screen but not in another.

the responses of these neurons were due not just to longer fixation on, for example, novel than familiar presentations of stimuli, for such novel object-place responses were found for only a small proportion of neurons. It was also noted that the places to which different neurons responded were found in all quadrants of the screen, so that the neuronal responses described here were not due to differential fixation of certain screen positions.

To test whether the neurons just described that responded to a combination of novelty and position would respond differently to novel and familiar stimuli in a task in which position was not important, the responses of 8 of these 24 neurons were also measured in a serial recognition memory task. It was found that 4 of these responded more to the novel than to familiar stimuli. The "memories" for stimuli of these neurons were relatively short, not exceeding 8 intervening stimuli.

Ten of these 24 neurons were also tested on a visual-discrimination task to control for the possibility of their different responses in the multiple serial object-place memory task being due to responses to "go" versus "no-go" or to reward. Only one neuron responded to "no-go," but this was $100 \mathrm{msec}$ later than its response to novel stimuli in the object-place task; thus, its

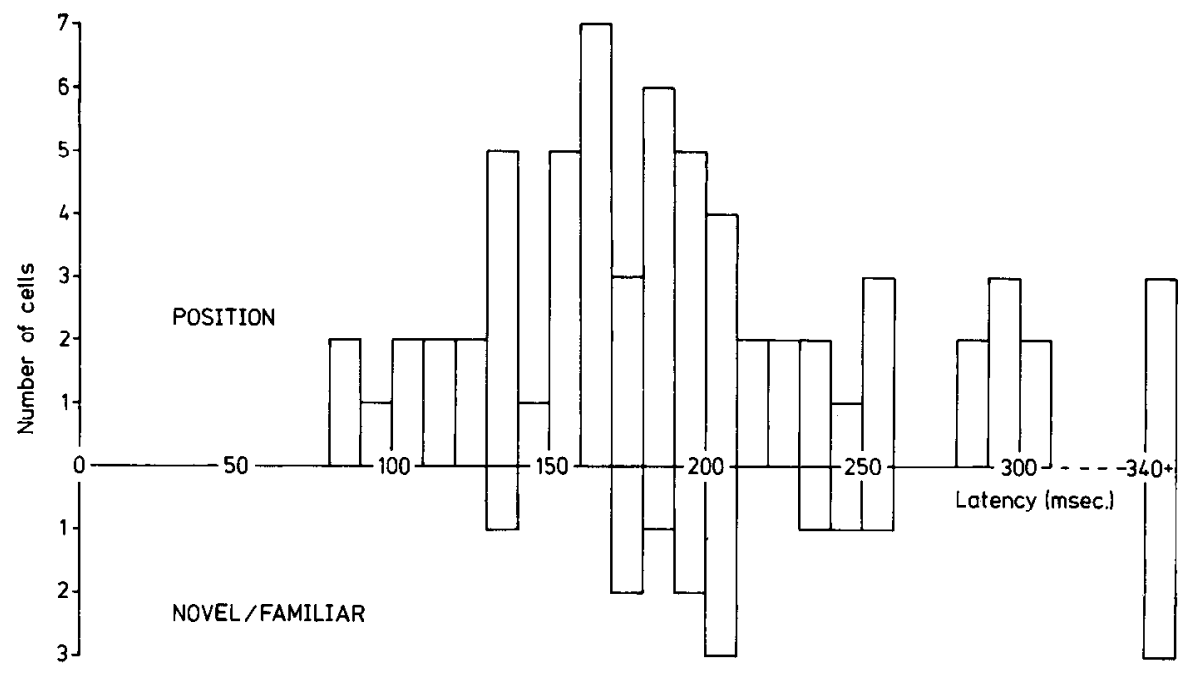

Figure 5. Response latencies of hippocampal formation neurons that responded differentially to different spatial positions (above) or to novel versus familiar visual stimuli (below). The differential response latencies are shown. 
A
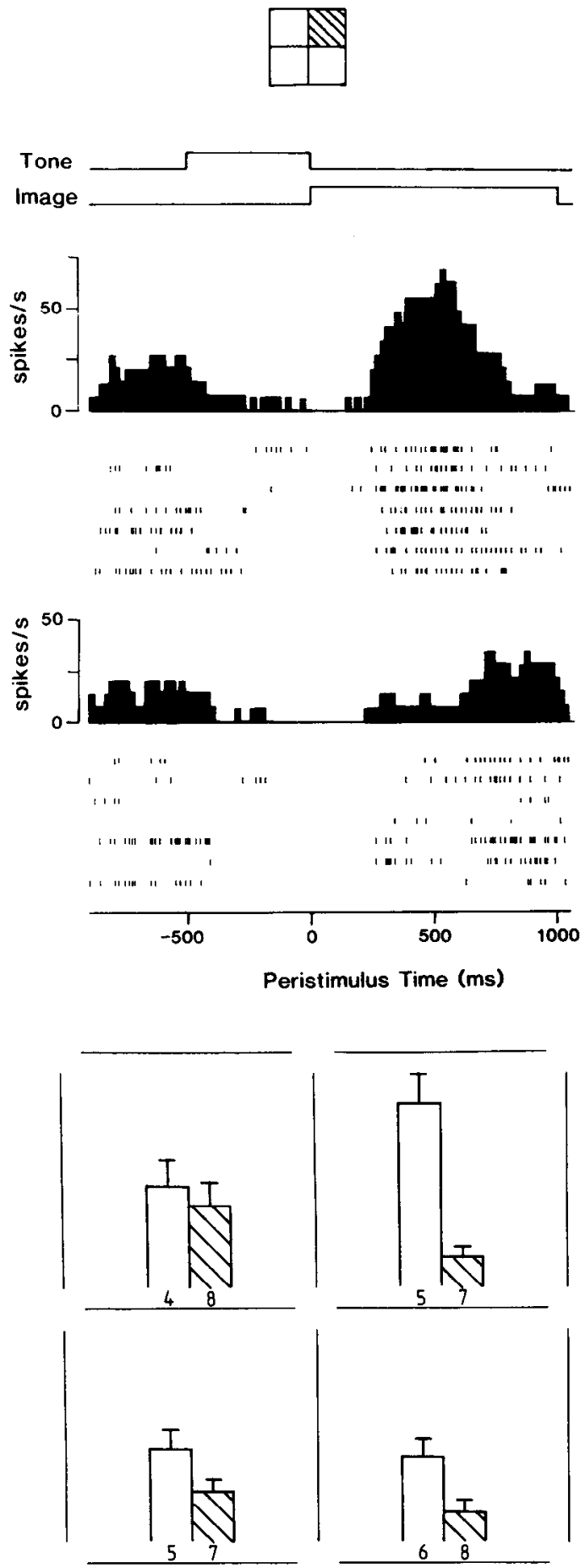

$[10$ spikes $/$ sec

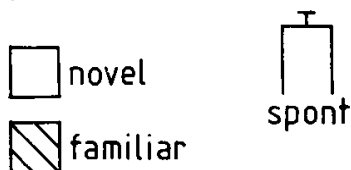

response to novel stimuli in the object-place task at $170 \mathrm{msec}$ was not due to the "no-go" trial contingency. Therefore, it is unlikely that any significant proportion of the responses of these hippocampal neurons in the object-place memory task can be

B
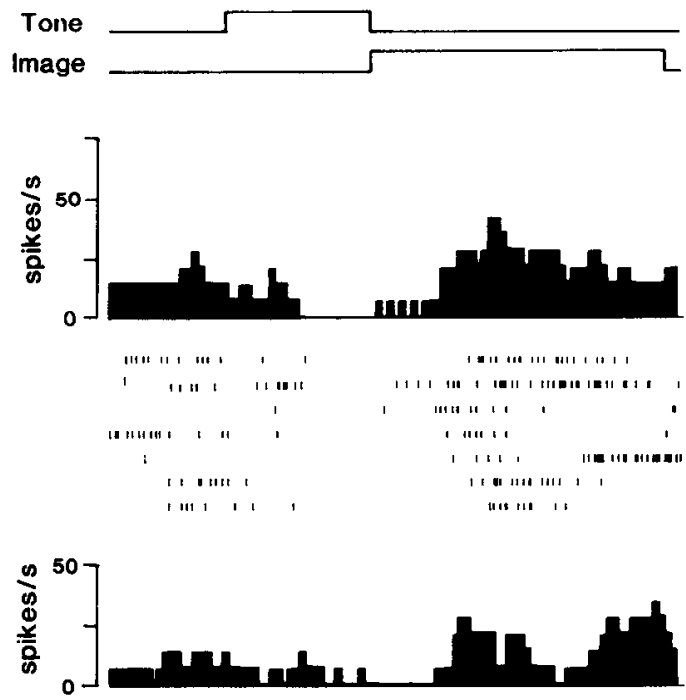

FAMILIAR

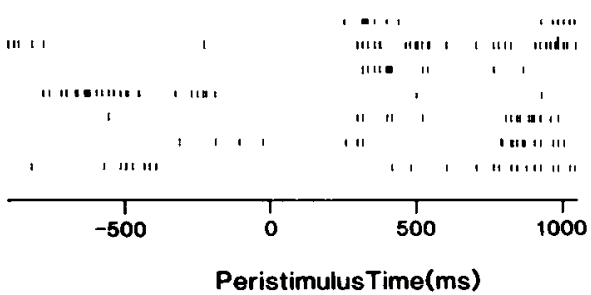

Figure 6. Top, Peristimulus rastergrams and time histograms of the activity of a hippocampal neuron when stimuli were presented for the first time (novel) or for the second time (familiar) in different positions $(A$ and $B)$. Bottom, Firing rate histograms of the activity of the same hippocampal neuron when stimuli were presented for the first time (novel) or for the second time (familiar) in different positions on the screen in the object-place memory task.

explained on the basis of the "go" or "no-go" requirement of the task.

Given that some cells in the hippocampus of the primate have spatial responses during the performance of an object-place 


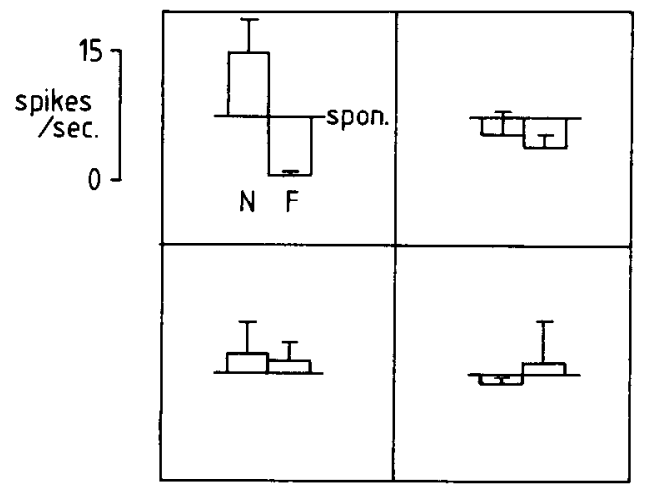

Figure 7. Firing rate histograms of the activity of a different hippocampal neuron when stimuli were presented for the first time (novel) or for the second time (familiar) in different positions on the screen in the object-place memory task.

memory task as just described, we next determined whether hippocampal neurons also had spatial responses in a simpler task in which stimuli appeared in one quadrant of the screen and the monkey could touch the stimulus while it was on for $1.0 \mathrm{sec}$ in order to obtain reward. The spatial stimuli and the spatial responses were thus very similar to those in the multiple object-place memory task, but there was no requirement to remember the stimuli and the position in which they had been shown. In this spatial touch task, it was found that 62 of 605 neurons analyzed $(10.2 \%)$ had spatial responses, that is, the firing rate was significantly different for different positions on the screen. Thus, it is not necessary for the monkey to perform an object-place memory task in order for hippocampal neurons to show spatial responses.

Given that some hippocampal neurons have spatial responses in these spatial tasks, it is next possible to consider whether this neuronal activity is related to the fact that stimuli were presented in different positions or to the spatial responses of reaching to one position on the screen as the monkey did in some of the tasks. Of 20 cells studied using the tasks that incorporated different stimulus presentations or different response requirements, it was found that $18(90 \%)$ responded in a spatial task in which there was no spatial response requirement. Thus, the majority of these neurons exhibited activities that were more closely related to sensory aspects of space than to spatial movements. I'wo neurons responded only in versions of these tasks that required movements to different positions in space. Thus, the activity of these neurons was related more to motor than to sensory aspects of the processing of spatial information.

For the 18 neurons just described, further information about the nature of the sensory-related spatial responsiveness is available. For example, the spatial responses of 5 cells were shown to depend not only on the quadrant of the screen on which the stimuli were presented, but also on the distance of the stimuli from the monkey. Further evidence that distance is a spatial property that is important in the responsiveness of at least some of these neurons was found in the clinical tests described below. In addition, 2 neurons had responses that depended not only on position in space, but also on the particular stimulus shown. The presence of neurons of this type was suspected on the basis of the responses obtained in the object-place memory task and was confirmed by additional tests in which the same set of stimuli was repeated a number of times in random order in each

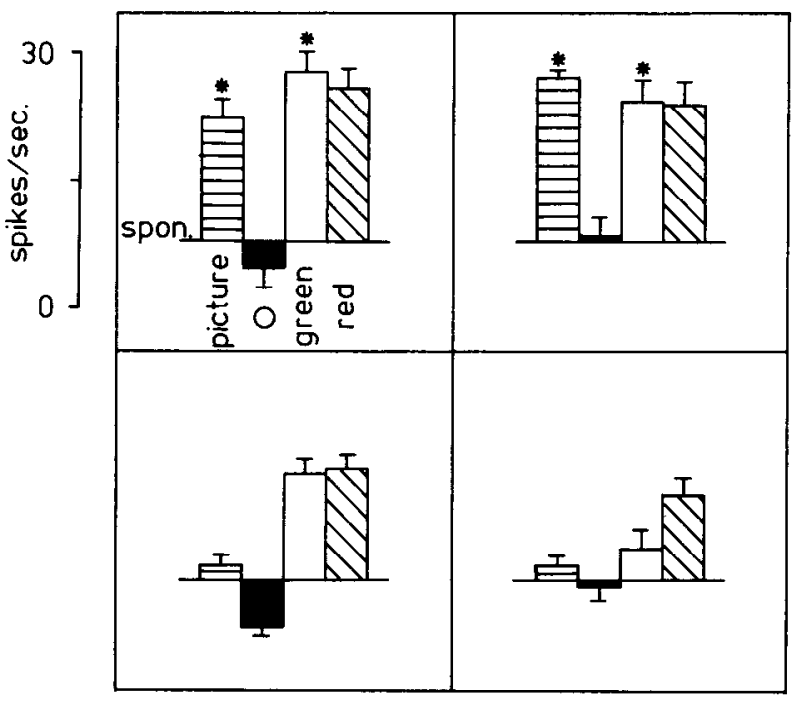

Figure 8. Firing rate histograms for a neuron that responded to a combination of particular visual stimuli and spatial position (see text). The stimuli shown on the screen were a pseudocolored image (picture), a white circle (O), a green square, or a red square. An ANOVA was performed to test for each stimulus whether different neuronal responses occurred when the stimulus was shown in different positions on the screen. The "picture" and the green stimulus both produced greater responses when shown in the upper half of the screen than when they were shown in the lower half $(*, p<0.01)$, that is, the response to these stimuli depended on the position in which they were shown.

position on the screen and the monkey was required to touch the stimuli. For example, the neuron shown in Figure 8 responded only to certain stimuli presented in the upper 2 quadrants of the screen (although the behavioral reach response was identical to all stimuli used). The 2 neurons of this type appeared to code for a combination of a class of stimulus with its position in space.

The locations of the neurons with spatial and related responses in the spatial tasks are plotted in Figure 9. The neurons with spatial responses were found in many different parts of the hippocampus and parahippocampal gyrus (Fig. 9). The activity recorded from the hippocampus proper could not easily be classified in terms of the "theta" versus "complex spike" types found in subprimate species such as the rat and rabbit (O'Keefe and Nadel, 1978). This is consistent with electroencephalographic studies in the primate showing a paucity of theta-like activity (e.g., Halgren et al., 1978, in human). We made a wider survey, including the present study, of the types of activity observed in more than 1500 neurons of the hippocampus and hippocampal formation. Only 22 neurons had activity of the complex spike type, and none of the differentially responsive neurons in the present study were of this type. We found 163 neurons that showed bursting activity, each burst containing between 2 and 6 spikes. Neurons with complex spike and bursting activity were almost exclusively confined to the hippocampus proper. The majority of neurons (1111) showed irregular firing activity (range of firing rate, $3-60 \mathrm{~Hz}$ ), and a proportion of these (241) showed occasional bursts of activity. Also, 79 neurons had rhythmic or phasic activity, showing clusters of short-duration action potentials. Each cluster typically lasted between $100-350 \mathrm{msec}$ of $100 \mathrm{~Hz}$ firing, with an intercluster interval of 100-400 msec. Many of these neurons were located in the dentate gyrus. Finally, 167 neurons displayed fairly regular firing activity that was neither bursting nor clustered. 


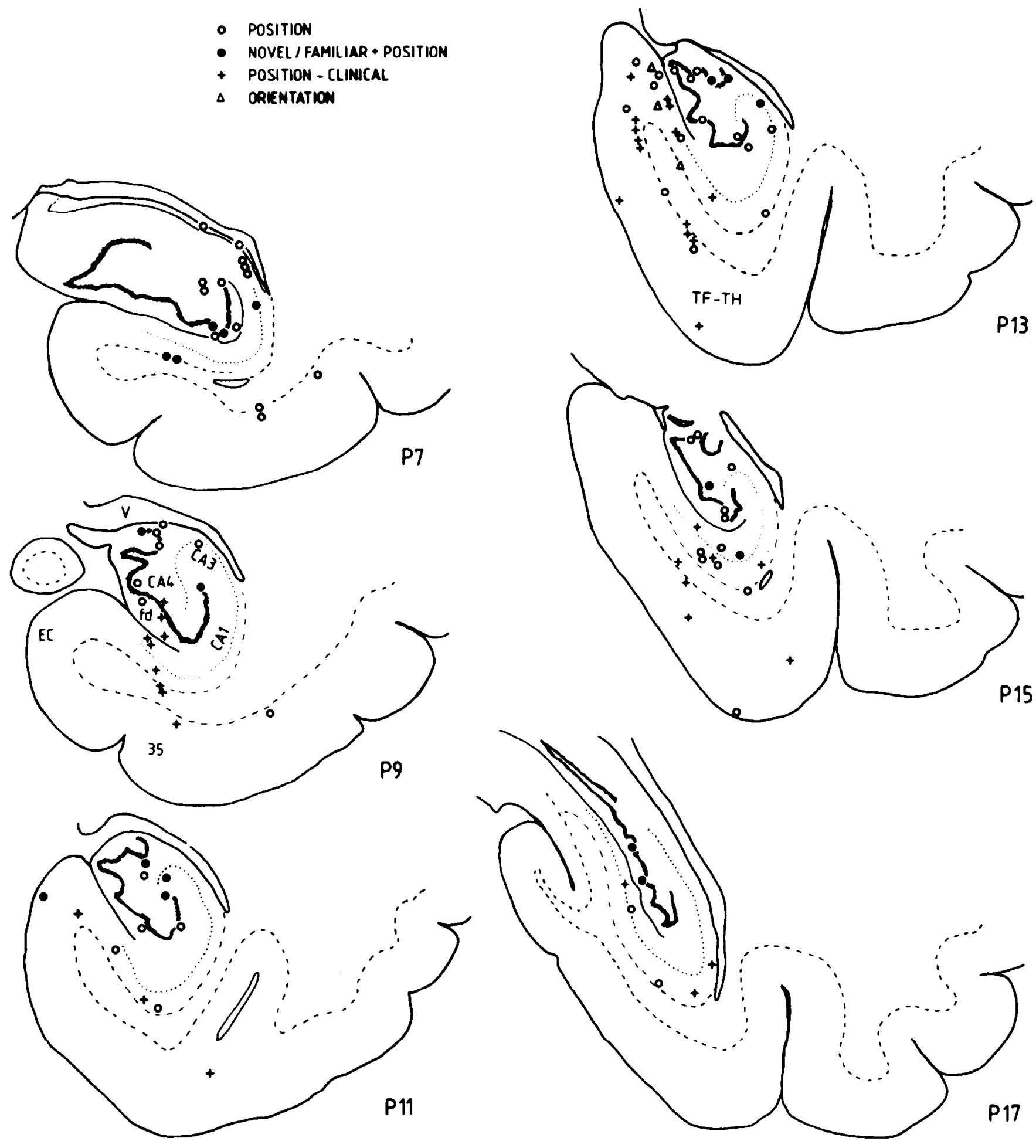

Figure 9. Recording sites in the hippocampus and the adjacent parahippocampal area of neurons with spatial responses (position), with responses dependent on whether a stimulus was scen in a particular position for the first time (novel/familiar position), with spatial responses demonstrated by "clinical" testing, and with responses related to the orientation of the monkey. CA1, CA3, CA4, hippocampal subfields (indicated by the dotted line); $E C$, entorhinal cortex; $f d$, fascia dentata; $T F-T H$, area in the parahippocampal gyrus; $V$, ventricle; 35 , perirhinal cortex. The boundary of the cortex with the white matter is indicated by the dashed line.

In the spatial tasks, it was found that $9.3 \%$ of the hippocampal neurons had responses to different positions on the screen, as described above. However, the screen filled only a part of the monkeys' visual space; moreover, the screen was usually at a fixed distance from the monkey. Thus, it is possible that if measurements had been made in more of the space around the monkey, many more cells with spatial responses might have been demonstrated. This was found to be the case in "clinical" 
tests in which the experimenter showed objects to the monkey in different parts of the laboratory. In this case, 105 neurons (25\% of those tested) were found to respond only when the monkey paid attention to some parts of the room. These neurons were probably a superset of those analyzed in the object-place memory task, for in a number of cases of neurons with spatial fields in the object-place memory task similar spatial fields were found when they were tested with an object such as a peanut held in the corresponding position in space in the laboratory with the video screen removed. The recording sites of these neurons are shown in Figure 9 (see "position-clinical"). In addition, 5 neurons were found that responded when the monkey was in a particular orientation in the room; interestingly, all were located in the subicular complex (see Fig. 9, “orientation"). Furthermore, 1 of 5 neurons tested responded when the chair was rotated, and this was direction selective. A number of cclls $(n=18)$ responded to the screcn in various ways, cither to its movement towards or away from the monkey.

\section{Discussion}

These results show that some neurons in the hippocampus of the monkey can respond differentially with respect to the place in which a stimulus is shown. The results were obtained in a task impaired by hippocampal damage in which the monkey had to remember the positions in which particular stimuli were seen (Mishkin, 1982; Gaffan and Saunders, 1985). Performance of a similar task is impaired in humans with right temporal lobe damage (Smith and Milner, 1981). The proportion of hippocampal neurons with spatial responses in these tasks was 93 of 994 analyzed, or $9.3 \%$. Many of these neurons had spatial responses that were highly significant, and significant or very highly significant results in the ANOVA were much more frequent than would be predicted by chance (as tested by a $\chi^{\prime}$ test: $\chi^{\top}=$ $27.8, d f=2, p \ll 0.001)$. It may be noted that in the tasks only a limited part of space was utilized and that more neurons in the monkey hippocampus might be shown to have spatial responses if the space utilized was more extensive. Indeed, in the clinical testing in which objects were shown throughout the experimental room, $25 \%$ of neurons tested had some spatial selectivity.

This is the first study we know of in which hippocampal neurons with responses related to the place in which a stimulus is shown have been demonstrated in the primate hippocampus. Watanabe and Niki (1985) measured the activity of hippocampal neurons in a delayed response task in which a red stimulus was shown on the right or the left; a delay period followed in which the monkey had to continue holding a central observing response lever, and then when the left and right lights illuminatcd simultaneously, the monkey could press the light that had previously been illuminated in order to obtain reward. In this task, some neurons $(2.2 \%)$ responded differentially to the left and right sample stimuli or in the delay which followed. As the monkey can prepare his response as soon as the sample stimulus is shown in this task (consistent with it being designated a delayed response task), and may even make mediating responses in the task such as leaning to the left or right as soon as the sample stimulus is shown, it is not clear whether the neurons with differential activity reflected the side on which the stimulus was shown, preparation for a motor response to that side, or even a mediating motor response made in the task. In the present object-place memory task, it is extremely unlikely that the neuronal activity reflected preparation of a motor response or a mcdiating motor response for the following reasons. First, when a stimulus was shown in a given position, the monkey could not code for any motor response at that time, but had to wait for up to 6 trials (16 in the $3 \times 3$ version) before he could determine whether to make a response. (His decision had to be based on whether he had seen a given stimulus in the same position in the preceding 6 (or 16) trials.) Mediating responses in such a situation are most unlikely. Second, the response made by the monkey in one version of the task was a nonspatial response, that of licking a tube in front of his mouth, so that the differential responses of the neurons to the different positions in which stimuli were shown could not have been due to a spatial response requirement of the task. Third, the majority of the neurons did not respond differentially to a novel, as compared with a familiar, object-place combination, so that differential motor responses are not likely to account for the differential responses of the neurons. Fourth, the responses of the neurons could still occur when a stimulus was shown in a particular position when the monkey was performing the task incorrectly or was not performing the task, or during clinical presentation of stimuli in certain positions, providing further evidence that the responses of these neurons are not related simply to motor responses. Fifth, the neurons did not respond in a visual-discrimination task in which the same motor response (of licking), and the same reinforcement (fruit juice) was given, providing further evidence that the motor response required or the reinforcement obtained cannot account for the responses of these hippocampal neurons. These points provide evidence that these hippocampal neurons in the monkey code for the place in which a stimulus is shown, rather than the response required to a particular spatial position.

The differential responses of these neurons to stimuli shown in different positions on the screen in a sense reflect different spatial response fields of these neurons. The question of whether these are retinotopically organized can best be addressed by further experiments in which the monkey is required to fixate different positions of the screen during the performance of the object-place memory task; in which he is required to make eye movements to small fixation spots in different positions on the screen; and in which stimuli are shown in different retinotopic positions when the monkey maintains fixation of different positions in space. Although these are new series of experiments which are suggested as a result of the findings described in this paper and are now being performed, we have noted in the results above that in the first new experiments of this type it has been found that the responses of the majority of neurons (at least 39 of a sample of 46) of the type described here are not due to eye movements per se, in that they do not occur when the monkey moves his eyes to fixate a small spot in different positions on the screen in a visual fixation task. We can add that for some neurons the responses occur when objects are shown in different spatial positions on the screen even when the monkey must continue fixating a small central spot. However, we think it unlikely that simple retinotopic coding accounts for the responses of these neurons, in that at least some of the neurons responded consistently when an object was shown in a particular position on the screen, even when, on some trials, the monkey fixated a different part of the screen. Further, some of the neurons responded differentially to novel, as compared with familiar, stimuli in a given position, and the responses of these neurons cannot be accounted for by a simple retinotopic mapping without additional processing. Also, it may be noted that 
the response properties of these neurons were very different from those of neurons in the retinotopically organized parts of the visual system such as the lateral geniculate nucleus or the striate cortex, where response latencies may be on the order of $30 \mathrm{msec}$, compared with 100-200 msec for the neurons in the hippocampus described here. The observation that the positions to which different neurons responded maximally were evenly distributed across the monitor screen indicates that the neuronal responses obtained were not due to differential visual stimulation by one part of visual space, but are instead consistent with the view that these neurons provide a representation of space. This is one of the main new findings described here, and it will be of interest in continuing investigations, of the type described above, to determine the coordinate system in which space is represented in the primate hippocampus. The size of the parts of space reflected in the responses of these neurons described here may well have been influenced by the task, but in that differential responses were shown across $12^{\circ}$ of visual space, the angle subtended by the monitor, the spatial fields of the neurons can be small compared with this angle.

These neurons may be compared with "place" cells recorded in the rat hippocampus (see O'Keefe, 1979). The "place" cells in the rat respond when the rat is in a particular place in the environment as specified by extramaze cues, whereas the cells described here respond to particular positions in space, or at lcast when stimuli arc shown in particular positions in space. The exact information that leads "place" cells in the rat to respond, and the nature of any mapping that may be being performed by the hippocampus, has not been easy to define (McNaughton et al., 1983). The present results in the monkey certainly do not prove that a "cognitive map" (O'Keefe and Nadel, 1978) is present in the hippocampus. However, by requiring different eye-fixation positions in the object-place memory task and by moving the monitor screen relative to the monkey's body, it should be possible to determine whether the neurons reflect information coded in retinotopic, egocentric, or allocentric space.

Although the response latencies of the neurons were not always sufficiently short for them to play a part in the performance of the object-place memory task, in some cases their responses did precede the responses made by the monkey in the objectplace memory task. This, together with the evidence that hippocampal lesions impair object-place memory tasks (Mishkin, 1982; Gaffan and Saunders, 1985), is consistent with the view that some of these neurons provide information useful in performing this task. The neurons with responses that occur independently of whether a stimulus is novel or familiar in a particular position may provide a useful spatial representation when a combination of a particular position and a particular stimulus must be represented in a working memory. The neurons with responses that depend on whether a stimulus has been seen before in each spatial location reflect the combination of position and novelty, and a population of neurons with information of this type distributed across it would contain the information necessary to perform the object and place memory task for which the hippocampus is required. We do not find it surprising that only a relatively small proportion of such neurons was found, for this would be required if many of the possible object and place combinations that the monkey might encounter were to be represented in a usefully discriminable form (see Rolls, 1987, 1988). As noted above, the presence of such neurons was detected in this investigation with high statistical reliability.
Finally, we note that only a proportion of hippocampal neurons had "space" responses and that other hippocampal neurons active in other tasks, such as delayed spatial response and conditional spatial response tasks, may also be important for functions performed by the hippocampus (Cahusac et al., 1989; Miyashita et al., 1989; Rolls, 1989).

\section{References}

Aggleton, J. P., and R. E. Passingham (1981) Stereotaxic surgery under $\mathrm{X}$-ray guidance in the Rhesus monkey, with special reference to the amygdala. Exp. Brain Res. 44: 271-276.

Brown, M. W. (1982) Effect of context on the response of single units recorded from the hippocampal region of behaviourally trained monkeys. In Neuronal Plasticity and Memory Formation, C. Ajmone Marsan and H. Matthics, cds., pp. 557-573, Raven, New York.

Bruning, J. L., and B. L. Kintz (1977) Computational Handbook of Statistics, 2nd ed., Scott, Foresman, Glenview, IL.

Cahusac, P. M. B., Y. Miyashita, and E. T. Rolls (1989) Responses of hippocampal formation neurons in the monkey related to delayed spatial response and object-place memory tasks. Behav. Brain Res. (in press).

Gaffan, D. (1987) Amnesia, personal memory and the hippocampus: Experimental neuropsychological studies in monkeys. In Cognitive Neurochemistry, S. M. Stahl, S. D. Iversen, and E. C. Goodman, eds., pp. 45-56, Oxford U. P., Oxford, UK.

Gaffan, D., and R. C. Saunders (1985) Running recognition of configural stimuli by fornix-transected monkeys. Q. J. Exp. Psychol. 37B: 61-71.

Halgren, E., T. L. Babb, and P. H. Crandall (1978) Human hippocampal formation EEG desynchronizes during attentiveness and movement. Electroencephalogr. Clin. Neurophysiol. 44: 778-781.

Judge, S. J., B. J. Richmond, and F. C. Chu (1980) Implantation of magnetic search coils for measurement of eye position: An improved method. Vision Res. 20: 535-538.

McNaughton, B. L., C. A. Barnes, and J. O'Keefe (1983) The contributions of position, direction, and velocity to single unit activity in the hippocampus of freely-moving rats. Exp. Brain Res. 52:41-49.

Merrill, E. G., and A. Ainsworth (1972) Glass-coated platinum-plated tungsten microelectrodes. Med. Biol. Eng. 10:662-672.

Milner, B. (1982) Some cognitive effects of frontal-lobe lesions in man. Phil. Trans. R. Soc. London [Biol.] 298: 211-226.

Mishkin, M. (1978) Memory in monkeys severely impaired by combincd but not scparatc removal of amygdala and hippocampus. Nature 273: 297-298.

Mishkin, M. (1982) A memory system in the monkey. Phil. Trans. R. Soc. London [Biol.] 298: 85-95.

Miyashita, Y., E. T. Rolls, P. M. B. Cahusac, H. Niki, and J. D. Feigenbaum (1989) Activity of hippocampal neurons in the monkey related to a stimulus-response association task. J. Neurophysiol. (in press).

Morris, R. G. M., P. Garrud, J. N. P. Rawlins, and J. O'Keefe (1982) Place navigation impaired in rats with hippocampal lesions. Nature 297: 681-683.

O'Keefe, J. (1979) A review of the hippocampal place cells. Prog. Neurobiol. 13: 419-439.

O'Keefe, J. (1983) Spatial memory within and without the hippocampal system. In Neurobiology of the IIippocampus, W. Seifert, ed., pp. 375-403, Academic, London.

O'Keefe, J., and L. Nadel (1978) The Hippocampus as a Cognitive Map, Clarendon, Oxford, UK.

Olton, D. S. (1983) Memory functions and the hippocampus. In $\mathrm{Neu}$ robiology of the Hippocampus, W. Seifert, ed., pp. 335-373, Academic, London.

Olton, D. S., J. T. Becker, and G. E. Handelman (1979) Hippocampus, space, and memory. Behav. Brain Sci. 2: 313-365.

Parkinson, J. K., E. A. Murray, and M. Mishkin (1988) A selective mnemonic role for the hippocampus in monkeys: Memory for the location of objects. J. Neurosci. 8: 4059-4167.

Rolls, E. T. (1987) Information representation, processing and storage in the brain: Analysis at the single neuron level. In The Neural and Molecular Bases of Learning, J.-P. Changeux and M. Konishi, eds., pp. 503-540, Wiley, New York.

Rolls, E. T. (1989) Functions of neuronal networks in the hippocampus 
and neocortex in memory. In Neural Models of Plasticity: Theoretical and Empirical Approaches, J. H. Byrne and W. O. Berry, eds., Academic, New York.

Rolls, E. T., M. J. Burton, and F. Mora (1976) Hypothalamic neuronal responses associated with the sight of food. Brain Res. 111: 53-66.

Rolls, E. T., M. K. Sanghera, and A. Roper-Hall (1979) The latency of activation of neurones in the lateral hypothalamus and substantia innominata during feeding in the monkey. Brain Res. 164: 121-135.

Rolls, E. T., D. I. Perrett, A. W. Caan, and F. A. W. Wilson (1982) Neuronal responses related to visual recognition. Brain 105: 611646.

Schwartz, E. L., R. Desimone, T. D. Albright, and C. G. Gross (1983) Shape recognition and inferior temporal neurons. Proc. Natl. Acad. Sci. USA 80: 5776-5778.
Scoville, W. B., and B. Milncr (1957) Loss of recent memory after bilateral hippocampal lesions. J. Neurol. Neurosurg. Psychiatry 20. $11-21$.

Smith, M. L., and B. Milner (1981) The role of the right hippocampus in the recall of spatial location. Neuropsychologia 19: 781-793.

Squire, L. R., and S. Zola-Morgan (1983) The neurology of memory: The case for man and non-human primate. In The Physiological Basis of Memory, J. A. Deutsch, ed., 2nd ed., Academic, New York.

Watanabe, T., and H. Niki (1985) Hippocampal unit activity and delayed response in the monkey. Brain Res. 325: 241-254.

Woodward, R. H., and P. L. Goldsmith (1964) Cumulative Sum Techniques: Mathematical and Statistical Techniques for Industry (ICI Monogr. No. 3), Oliver \& Boyd, Edinburgh. 